\title{
Cardamonin Regulates miR-21 Expression and Suppresses Angiogenesis Induced by Vascular Endothelial Growth Factor
}

\author{
Fu-Sheng Jiang, ${ }^{1}$ Sha-Sha Tian, ${ }^{1}$ Jin-Jian Lu, ${ }^{2}$ Xing-Hong Ding, ${ }^{1}$ Chao-Dong Qian, ${ }^{1}$ \\ Bin Ding, ${ }^{1}$ Zhi-Shan Ding, ${ }^{1}$ and Bo Jin ${ }^{1}$ \\ ${ }^{1}$ College of Life Sciences, Zhejiang Chinese Medical University, Hangzhou, Zhejiang 310053, China \\ ${ }^{2}$ State Key Laboratory of Quality Research in Chinese Medicine, Institute of Chinese Medical Sciences, University of Macau, Macau
}

Correspondence should be addressed to Bo Jin; jinbo@zcmu.edu.cn

Received 9 March 2015; Revised 30 June 2015; Accepted 5 July 2015

Academic Editor: Gagan Deep

Copyright (C) $2015 \mathrm{Fu}$-Sheng Jiang et al. This is an open access article distributed under the Creative Commons Attribution License, which permits unrestricted use, distribution, and reproduction in any medium, provided the original work is properly cited.

Cardamonin has promising potential in cancer prevention and therapy by interacting with proteins and modifying the expressions and activities, including factors of cell survival, proliferation, and angiogenesis. In our precious study, we have demonstrated that cardamonin suppressed vascular endothelial growth factor- (VEGF-) induced angiogenesis as evaluated in the mouse aortic ring assay. It is also known that microRNAs (miRNAs) play important roles in angiogenesis. Herein, we hypothesized whether antiangiogenesis effect of cardamonin in human umbilical vein endothelial cells (HUVECs) triggered by VEGF was associated with miRNAs. We found that cardamonin reduced the miR-21 expression induced by VEGF in HUVECs. Treatment with miR21 mimics abolished the effects of cardamonin on VEGF-induced cell proliferation, migration, and angiogenesis in HUVECs. However, treatment with miR-21 inhibitors presented the opposite effects, indicating the vital role of miR-21 in this process. Our study provides a new insight of the preliminary mechanism of anti-VEGF-induced angiogenesis by cardamonin in HUVECs.

\section{Introduction}

Cardamonin is a chalcone that belongs to the flavonoid family isolated from many kinds of herbs, such as Alpinia katsumadai, Carya cathayensis Sarg. [1], G. biloba [2], and Gynostemma pentaphyllum [3], and is often responsible for the yellow pigmentation of plants $[4,5]$. As shown by the increasing number of publications, cardamonin has attractive scientists' attention due to its benefits to human health. It presents various pharmacological activities, including anti-inflammatory $[6,7]$, antineoplastic [8], antioxidant [9], and anti-infectious [10] properties. Cardamonin inhibits smooth muscle cell proliferation and migration [11, 12], prevents endothelial barrier dysfunction [13], and suppresses vascular endothelial growth factor- (VEGF-) induced angiogenesis as evidenced by the mouse aortic ring assay [14].

Excessive angiogenesis (neovascularization) is the characteristic of a number of serious diseases, including cancer [15], rheumatoid arthritis [16], and atherosclerosis [17]. In our previous study, we found the total flavonoids (TFs), isolated from the leaves of Carya cathayensis Sarg., and five flavonoids of the main components of the TFs, that is, cardamonin, pinostrobin chalcone, wogonin, chrysin, and pinocembrin, could suppress VEGF-induced angiogenesis as detected in the mouse aortic ring assay, and cardamonin showed the strongest inhibitory activity. Our further study demonstrated cardamonin remarkably suppressed VEGF-induced proliferation and migration, by decreasing the phosphorylation of ERK and AKT induced by VEGF with a dose-dependent manner in HUVECs [14]. Although these studies presented interpretation for cardamonin-mediated antiangiogenesis, deeper insights for the mechanisms involved are still appreciated.

MicroRNAs (miRNAs) are a new class of small nonprotein-coding RNAs which are approximately 19-22 bases in length and that control gene expression posttranscriptionally by binding to various mRNA targets, predominantly in the $3^{\prime}$ untranslated region [18]. They control a wide range of 
TABLE 1: The list of primers used in qRT- PCR.

\begin{tabular}{lcc}
\hline & Sense primers & Antisense primers \\
\hline miR-17-5p & CAAAGTGCTTACAGTGCAGGTAG & \\
miR-19a & TGTGCAAATCTATGCAAAACTGA & Provided by miScript SYBR Green \\
miR-23a & CACATTGCCAGGGATTTCC & PCR Kit (TaKaRa, Dalian, China) \\
miR-24 31 & GCTCAGTTCAGCAGGAACAGA & \\
miR-34a & GCAAGATGCTGGCATAGCT & \\
miR-126 & GGCAGTGTCTTAGCTGGTTGT & \\
miR-130a & TCGTACCGTGAGTAATAATGCG & \\
miR-132 & CAGTGCAATGTTAAAAGGGCAT & \\
miR-16 & TAACAGTCTACAGCCATGGTCG & TAGCAGCACGTAAATATTGGG \\
RNU6B & ACGTTGTGTAGCTTATCAGTG & AACGCTTCACGAATTTGCGT \\
\hline
\end{tabular}

biological functions such as cell proliferation, differentiation, apoptosis, and angiogenesis in endothelia cells [1921]. These reports indicate that miRNAs might be involved in cardamonin-induced regulation of angiogenesis. In the present study, we investigated whether or not miRNAs have potential effects on cardamonin-induced inhibition of proliferation, migration, and tube formation in HUVECs induced by VEGF.

\section{Materials and Methods}

2.1. Cell Culture and Chemicals. HUVECs were purchased from Shanghai Institute of Cell Biology at the Chinese Academy of Sciences (Shanghai, China), cultured in RPMI1640 (Gino Biomedical Technology, Hangzhou, Zhejiang, China), and supplemented with $10 \%$ heat inactivated fetal bovine serum (Tianhang Biological Technology, Huzhou, Zhejiang, China). All cells were incubated at $37^{\circ} \mathrm{C}$ in humidified $5 \% \mathrm{CO}_{2}$ and $95 \%$ air atmosphere. Cells at passages 3 to 8 were used in all experiments. Cardamonin was purchased from the National Institute for the Control of Pharmaceutical and Biological Products (Beijing, China).

2.2. Choosing miRs for Screening. Recent literatures revealed that a total of $17 \mathrm{miRs}$ and $1 \mathrm{miR}$ cluster had been reported to have connections with endothelial functions [22, 23]. We selected 14 of these miRs which might have been involved in regulation of angiogenesis, including miR-17-5p, miR-19a, miR-23a, miR-24, miR-31, miR-34a, miR-126, miR-130a, miR132, miR-16, miR-21, miR-217, miR-221, and miR-378 for our study. Among them, miR-217, miR-221, and miR-378 were excluded out from our further work due to the nonspecificity of the primers that were used in the quantitative RT-PCR (qRT-PCR).

2.3. $q R T-P C R$. After being incubated with $8 \mathrm{ng} / \mathrm{mL}$ VEGF, HUVECs were treated with $50 \mu \mathrm{M}$ cardamonin for $3 \mathrm{~h}, 6 \mathrm{~h}$, and $24 \mathrm{~h}$, respectively. The total RNA was extracted with Trizol (Invitrogen, Carlsbad, California, USA), using the standard method. cDNA synthesis was performed with $1 \mu \mathrm{g}$ of total RNA, using the One Step PrimeScript miRNA cDNA
Synthesis Kit (TaKaRa, Dalian, China) according to the manufacturer's instructions. qRT-PCR was performed on the ABI 7500 cycler (Applied Biosystems, Foster city, California, USA), using the miScript SYBR Green PCR Kit (TaKaRa, Dalian, China) according to the manufacturer's protocol. RNU6B (U6) was used as the endogenous control. The primers were designed using the untranslated sequences at $5^{\prime}$ and $3^{\prime}$ region which can be found in Table 1 . The antisense primers were provided by miScript SYBR Green PCR Kit (TaKaRa, Dalian, China).

2.4. Small RNA Transfection. HUVECs were seeded for $24 \mathrm{~h}$ prior transfection when they reached $60-80 \%$ confluence. miR-21 inhibitors or miR-21 mimics, obtained from Ambion (Austin, Texas, USA), were transfected into HUVECs with the Lipofectamine RNAiMAX Reagent (Invitrogen, Carlsbad, California, USA) at a final concentration of $30 \mathrm{nM}$. A nonsilencing siRNA was used as a negative control. Cells were incubated with small RNA complexes for $24 \mathrm{~h}$ before the medium was changed.

2.5. Cell Proliferation Assay. HUVECs in 96-well plate were transfected with miR-21 mimic, miR-21 mimic negative control, miR-21 inhibitor, and miR-21 inhibitor NC and were allowed to grow for $24 \mathrm{~h}$. After medium was changed, VEGF $(8 \mathrm{ng} / \mathrm{mL})$ [14] was added into the wells with or without $50 \mu \mathrm{M}$ cardamonin except for the control group, and the incubation was continued for another $24 \mathrm{~h}$. Cell proliferation was assayed by 3-(4,5-dimethylthiazol-2-yl)-5-(3-carboxymethoxyphenyl)-2-(4-sulfophenyl)-2H-tetrazolium (MTS, Promega, Beijing, China) test according to the manufacturer's protocol. The absorbance at $460 \mathrm{~nm}$ was determined with a microplate reader (Bio-Rad, San Francisco, California, USA). For each group, 5 duplicate wells were detected per experiment. Cell nuclei were stained with Hoechst 33342 and observed with an inverted fluorescent microscope (Olympus, Tokyo, Japan).

2.6. Wound Healing Assay. An in vitro wound healing assay was performed to measure the unidirectional migration in 
TABLE 2: Expression levels of miRNAs after cardamonin treatment (means \pm SD).

\begin{tabular}{|c|c|c|c|c|c|}
\hline \multicolumn{2}{|c|}{ Treatment } & \multirow{2}{*}{$\mathrm{miR}$} & \multicolumn{3}{|c|}{ Fold change } \\
\hline VEGF & Car & & $3 \mathrm{~h}$ & $6 \mathrm{~h}$ & $24 \mathrm{~h}$ \\
\hline+ & - & & 1.00 & 1.00 & 1.00 \\
\hline+ & + & miR-17-5p & $1.31 \pm 0.45$ & $0.96 \pm 0.32$ & $0.45 \pm 0.10^{* *}$ \\
\hline+ & + & miR-19a & $0.85 \pm 0.15$ & $0.70 \pm 0.23^{*}$ & $0.32 \pm 0.06^{* *}$ \\
\hline+ & + & miR-23a & $1.88 \pm 0.69^{*}$ & $0.50 \pm 0.12^{* *}$ & $0.35 \pm 0.13^{* *}$ \\
\hline+ & + & miR-24 & $0.75 \pm 0.18$ & $0.46 \pm 0.17^{* *}$ & $0.42 \pm 0.06^{* *}$ \\
\hline+ & + & miR-31 & $1.33 \pm 0.53$ & $0.78 \pm 0.12^{*}$ & $0.36 \pm 0.11^{* *}$ \\
\hline+ & + & miR-34a & $1.19 \pm 0.62$ & $0.50 \pm 0.09^{* *}$ & $0.43 \pm 0.07^{* *}$ \\
\hline+ & + & miR-126 & $1.23 \pm 0.65$ & $0.55 \pm 0.06^{* *}$ & $0.65 \pm 0.20^{*}$ \\
\hline+ & + & miR-130a & $0.93 \pm 0.48$ & $0.52 \pm 0.17^{* *}$ & $0.72 \pm 0.26^{*}$ \\
\hline+ & + & miR-132 & $1.10 \pm 0.59$ & $0.61 \pm 0.17^{* *}$ & $0.39 \pm 0.06^{* *}$ \\
\hline+ & + & miR-16 & $1.29 \pm 0.61$ & $0.63 \pm 0.19^{* *}$ & $0.37 \pm 0.09^{* *}$ \\
\hline+ & + & miR-21 & $1.11 \pm 0.62$ & $0.42 \pm 0.16^{* *}$ & $0.29 \pm 0.12^{* *}$ \\
\hline
\end{tabular}

Note: ${ }^{* * * *} p<0.05$ and 0.01 , respectively, compared with the VEGF group. miRNA level was determined by qRT-PCR after HUVECs were treated with VEGF and cardamonin for 3, 6, and 24 hours. Data was expressed as fold change of VEGF group.

HUVECs. HUVECs $\left(2 \times 10^{4}\right.$ cells $\left./ \mathrm{mL}\right)$ were seeded into 24-well plates. Cells were allowed to grow for $24 \mathrm{~h}$ after being transfected with miR-21 mimic, miR-21 mimic negative control (NC), miR-21 inhibitor, and miR-21 inhibitor NC RNAs and the monolayers of HUVECs were scratch-wounded to a $1 \mathrm{~mm}$ depth in a straight line using a $10 \mu \mathrm{L}$ pipette-tip when cells were at $70-80 \%$ confluence. VEGF $(8 \mathrm{ng} / \mathrm{mL})$ was added into the medium with or without $50 \mu \mathrm{M}$ cardamonin, and the incubation was continued for another $24 \mathrm{~h}$. Images were photographed both immediately after wounding and after $24 \mathrm{~h}$ incubation, using a phase-contrast microscope (Olympus, Tokyo, Japan). Migration was calculated as the area of HUVECs that migrated from the injured edge into the wound zone. At least four points in each of three random fields were examined for each of three independent wounds.

2.7. Tube Formation. Tube formation by endothelial cells is a key step in angiogenesis which has several types of cells such as endothelia cells and pericytes participate [24]. Therefore, we investigated how cardamonin regulates capillary tube formation by HUVECs. HUVECs were cultured and transfected as described above. $24 \mathrm{~h}$ after transfection, cells were harvested and diluted to $2 \times 10^{5}$ cells $/ \mathrm{mL}$ in $0.5 \%$ FBS containing $20 \mathrm{ng} / \mathrm{mL}$ VEGF [25] and with or without $50 \mu \mathrm{M}$ cardamonin and seeded into matrigel-coated 24-well plate. After being cultured at $37^{\circ} \mathrm{C}$ for $8 \mathrm{~h}$, the branch points of the capillarylike tubes were counted under a light microscopy (Olympus, Tokyo, Japan) at 100 magnification for three random fields [25].

2.8. Statistical Analysis. Data were given as mean \pm SD. Statistical analysis was performed using one-way ANOVA and Dunnett's post-hoc test, with $p<0.05$ being considered to indicate statistical significance.

\section{Results}

3.1. miR-21 Expression Was Downregulated in HUVECs Induced by VEGF after Cardamonin Treatment. The expression of 11 miRs associated with angiogenesis was quantitatively analyzed after treatment with VEGF $(8 \mathrm{ng} / \mathrm{mL})$ and cardamonin $(50 \mu \mathrm{M})$ for $3 \mathrm{~h}, 6 \mathrm{~h}$, or $24 \mathrm{~h}$, respectively. We found that cardamonin suppressed the expression for most of the miRs at $6 \mathrm{~h}$ and $24 \mathrm{~h}$ (Table 2). As noted, miR-21 was suppressed the most among these 11 miRs (with 58\% inhibition compared with VEGF alone) (Table 2). The suppression was further down by $71 \%$ at $24 \mathrm{~h}$ compared with VEGF alone as determined by qRT-PCR. Other miRNAs, such as miR-23a, miR-31, miR-132, or miR-16, were also significantly downregulated with cardamonin treatment for $24 \mathrm{~h}$ compared to that of treatment for $3 \mathrm{~h}$.

3.2. Transfection with miR-21 Mimics or Inhibitors Aggravates or Attenuates the Intracellular Level of miR-21. Since most of miRs have been downregulated and miR-21 was strongly suppressed by cardamonin, we used miR-21 mimics and miR21 inhibitors to test the function of cardamonin on HUVECs. As shown in Figure 1, expression of miR-21 of HUVECs was augmented when cells were transfected with $30 \mathrm{nM}$ miR-21 mimics, while expression of miR-21 was significantly reduced when transfected with $30 \mathrm{nM}$ miR-21 inhibitors. None of the negative control RNAs had a significant effect.

3.3. Cardamonin-Mediated Inhibition of HUVECs Proliferation through the Expression of miR-21. The cell viability was detected by the MTS test, which can reflect the proliferative ability of the HUVECs stimulated by VEGF. When cardamonin was added into the medium, HUVECs viability was significantly reduced (Figure 2 ). To test whether the inhibitory effect was associated with miR-21, miR-21 inhibitor was added together with cardamonin. Lower expression level 


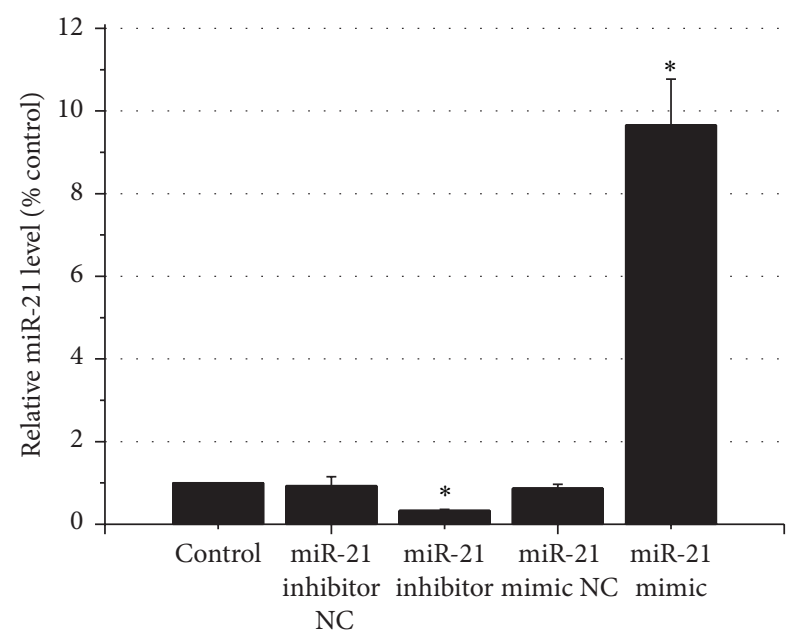

FIGURE 1: Relative miR-21 levels after transfection. Note: relative miR-21 lever was determined $24 \mathrm{~h}$ after transfection. ${ }^{*} p<0.01$ as compared with the control.

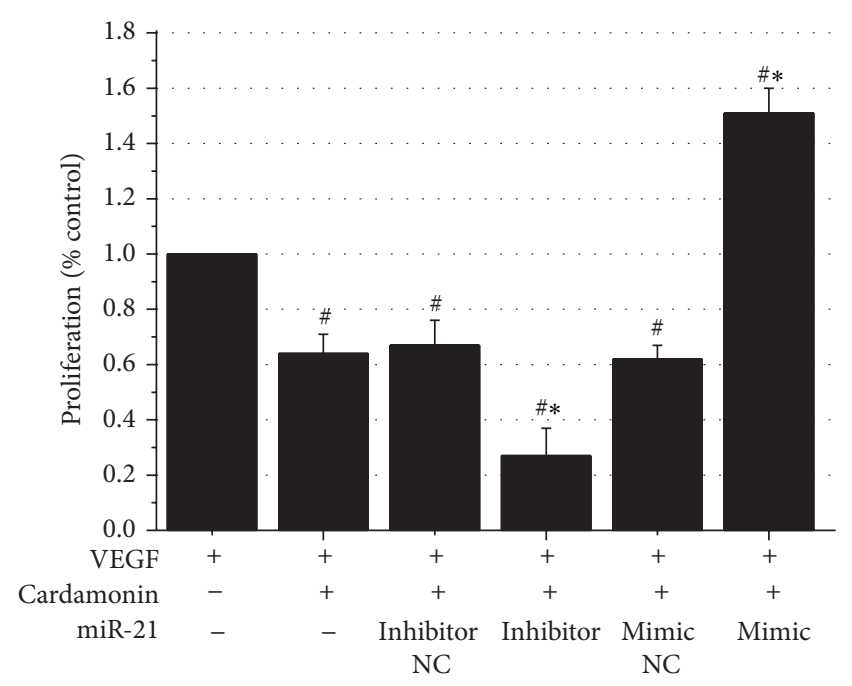

FIgURE 2: Cardamonin-mediated inhibition of HUVECs proliferation (means $\pm \mathrm{SD}$ ). Note: HUVECs were treated with VEGF (control), VEGF + cardamonin $(50 \mu \mathrm{M})$, or VEGF + cardamonin $(50 \mu \mathrm{M})+$ miRs. ${ }^{*} p<0.01$ as compared with the VEGF group, ${ }^{*} p<0.01$ as compared with the VEGF + cardamonin group.

of miR-21 caused a significantly stronger inhibition of HUVECs proliferation by $57.8 \%$ compared with cardamonin alone. To test whether cardamonin' inhibitory effect could be stopped by increasing the expression of miR-21, miR21 mimic was given together with cardamonin. As show in Figure 2, cardamonin's inhibition was completely inversed. HUVECs proliferation was $151.0 \%$ of VEGF. These data suggested that cardamonin inhibited HUVECs by downregulation of miR-21. The excessive expression of miR-21 induced by transfection of miR-21 completely eliminated cardamonin's action and showed a higher proliferation above its basal level (stimulated by VEGF). The negative control RNAs for both the mimic and the inhibitor had no significant effects on cell proliferation (Figure 2).

3.4. Inhibitory Effect of Cardamonin on Endothelial Migration in HUVECs Associated with Downregulation of the Expression Level of miR-21. In previous study, we found that cardamonin significantly inhibited HUVECs migration [14]. The migration of HUVECs was examined with a wound healing assay in this study. As shown in Figure 3, cardamonin significantly inhibited HUVECs migration by $65 \%$. Because cardamonin caused downregulation of miR-21, we hypothesized that this inhibition was associated with the expression of miR21. When miR-21 expression was decreased by transfection of miR-21 inhibitors, the migration inhibition was further downregulated by another $12.6 \%$. When miR-21 expression was upregulated by mimic treatment, the mobility of HUVECs increased significantly by $15.4 \%$. Neither the miR- 21 mimic NC nor miR-21 inhibitor NC had a significant effect on the regulation of cardamonin-inhibited HUVECs mobility (Figure 3). To understand the effect of miR-21 on HUVECs migration, miR-21 mimic and miR-21 inhibitor were transfected; the results indicated that miR-21 overexpression can significantly increase HUVECs migration (118.2\%) induced by VEGF; otherwise, HUVECs migration was remarkably inhibited by $26.4 \%$, which confirmed the importance of miR21 on HUVECs migration.

3.5. Inhibitory Effect of Cardamonin on Tube Formation in HUVECs Associated with Downregulation of the Expression of miR-21. New capillary formation is required for the initial steps of angiogenesis, which involves processes such as endothelial cell proliferation and migration [26]. To investigate whether the inhibitory effects of cardamonin is associated with miR-21 expression, we evaluated the effects of cardamonin on VEGF-induced tube formation in HUVECs after being transfected with miR-21 mimic. Transfection with miR21 mimic significantly stimulated the formation of capillarylike structures, when compared to VEGF treatment alone, and this action was significantly suppressed by transfection with miR-21 inhibitor alone; these data suggested that miR-21 plays an important role during angiogenesis. However, cardamonin treatment alone could also significantly suppress the formation of capillary-like structures by HUVECs, as compared to VEGF treatment alone, and cardamonin cotreatment with miR-21 inhibitor could further inhibit tube formation, and this could be remarkably reversed by cotreatment cardamonin with miR-21 mimic. Neither the miR-21 mimic NC nor the inhibitor NC had a significant effect on the regulation of cardamonin-inhibited tube formation (Figure 4). These data reconfirmed that cardamonin inhibits tube formation in HUVECs associated with downregulation of the expression of miR-21.

\section{Discussion}

Angiogenesis is a complex process of generating new capillary blood vessels. Regulation of angiogenesis includes endothelial cell proliferation, migration, basement membrane breakdown, and couple growth factors involved such as VEGF and 

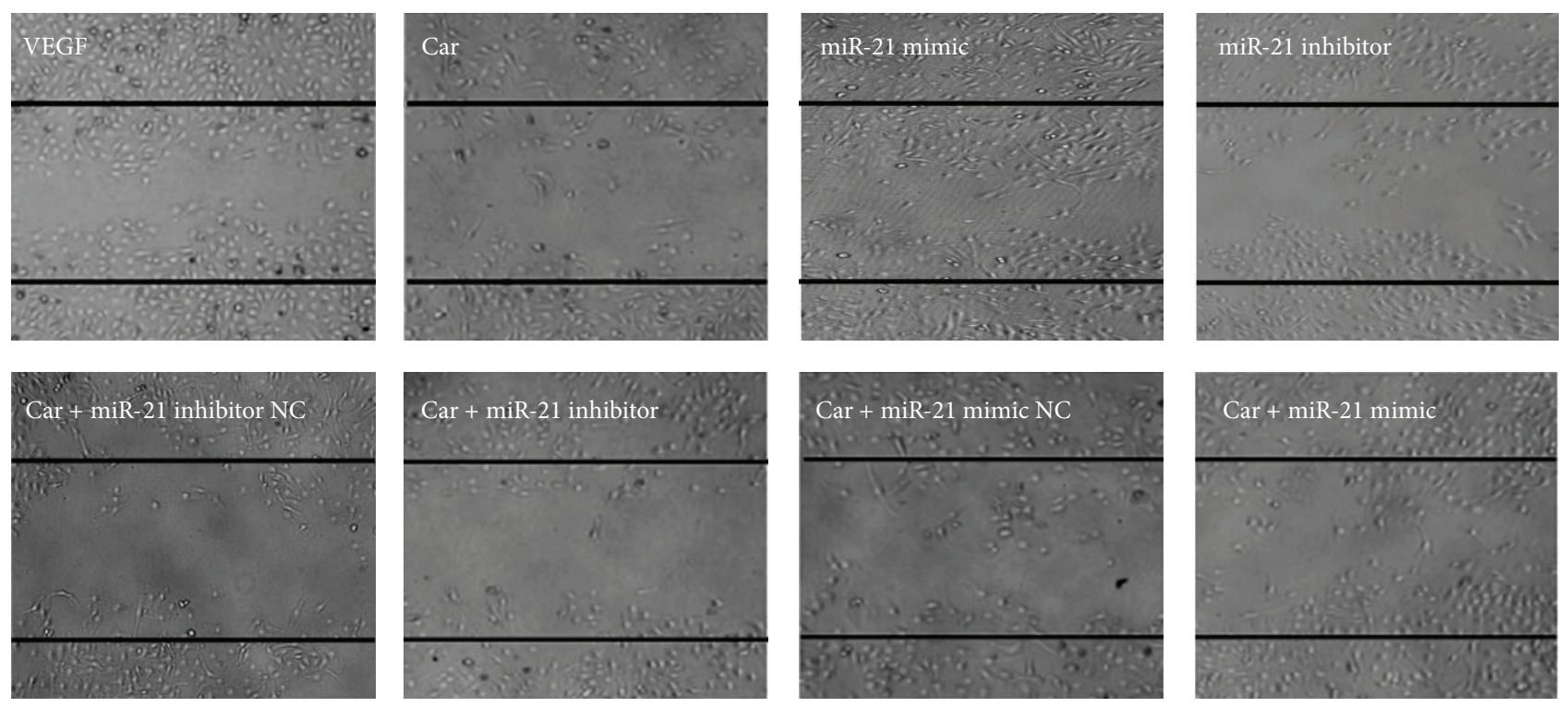

(a)

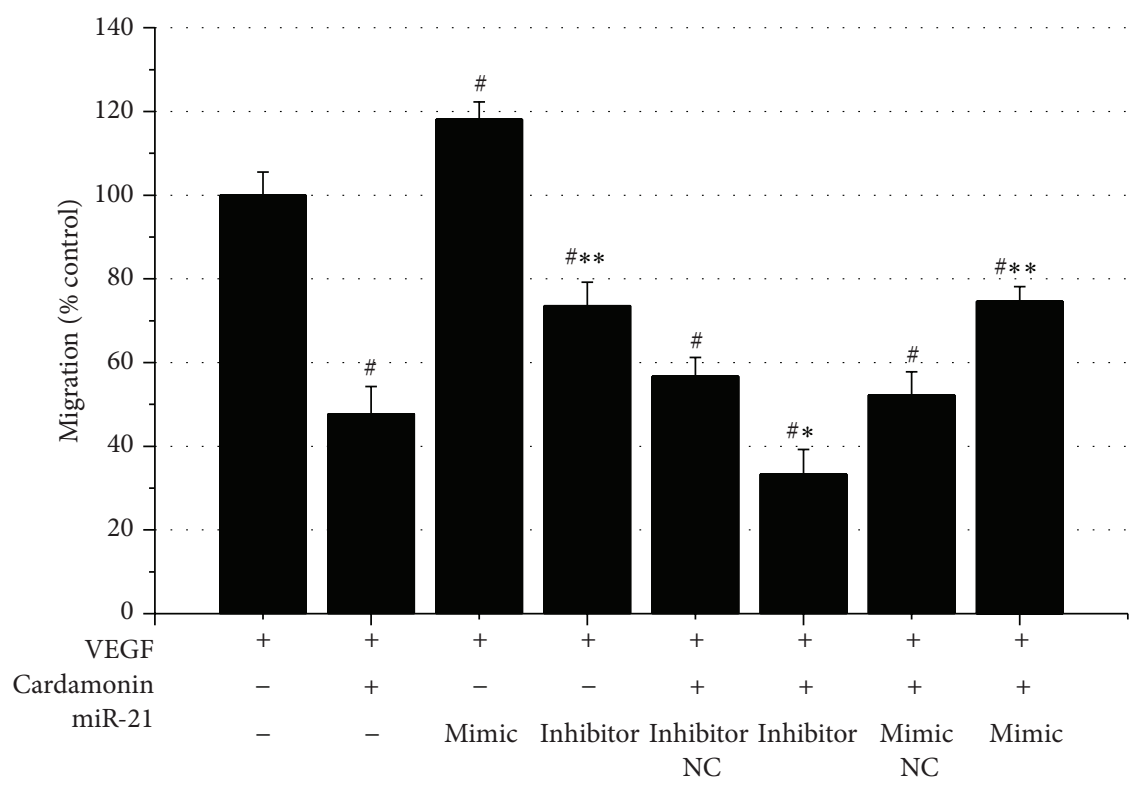

(b)

FIGURE 3: Cardamonin-mediated inhibition of HUVECs migration. Note: HUVECs in a six-well plate were photographed at the $0 \mathrm{~h}$ and $24 \mathrm{~h}$ time points. The areas of cells that migrated to the wound zones were measured separately with Image-Pro Plus 6.0 software. The experiment was repeated 3 times. (a) Cells in a six-well plate were photographed at $24 \mathrm{~h}$ time points. (b) The areas of cells that migrated into the wound zones, after treated with VEGF (control), VEGF + miR-21s, VEGF + cardamonin, or VEGF + cardamonin + miR-21s. ${ }^{\#} p<0.01$ as compared with the VEGF group, ${ }^{* * *} p<0.05$ and 0.01 , respectively, as compared with the VEGF+ cardamonin group.

FGF-2. Inhibition of endothelial cell proliferation and migration might contribute to the antiangiogenic activity. In our previous study, we found cardamonin remarkably suppressed VEGF-induced angiogenesis as detected in the mouse aortic ring assay. Cardamonin also obviously suppressed proliferation and migration induced by VEGF with a dose-dependent manner in HUVECs [14]. miRs are short noncoding RNAs function as negative regulators of gene expression which have been regulators of angiogenesis. Herein, we found for the first time that miR-21 was downregulated strikingly after treatment with cardamonin $(50 \mu \mathrm{M})$ in a time dependent manner.

As miR-21 is frequently overexpressed in human cancers and acts as oncogene and is involved in promoting cell proliferation, invasion, and migration [27-29], it may also play pivotal role in endothelial cell proliferation and migration and influence angiogenesis. Guduric-Fuchs et al. reported that downregulation of $\mathrm{miR}-21$ reduced the proliferation, 

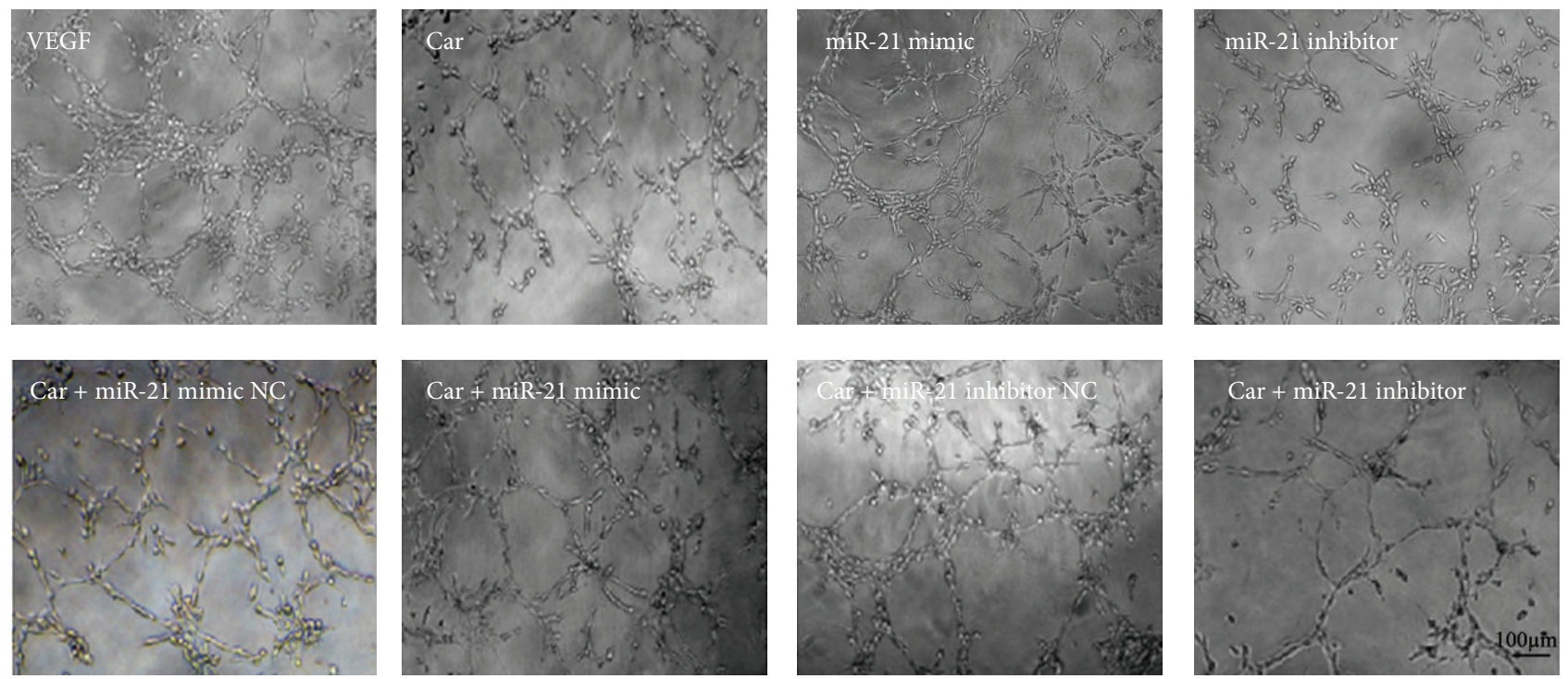

(a)

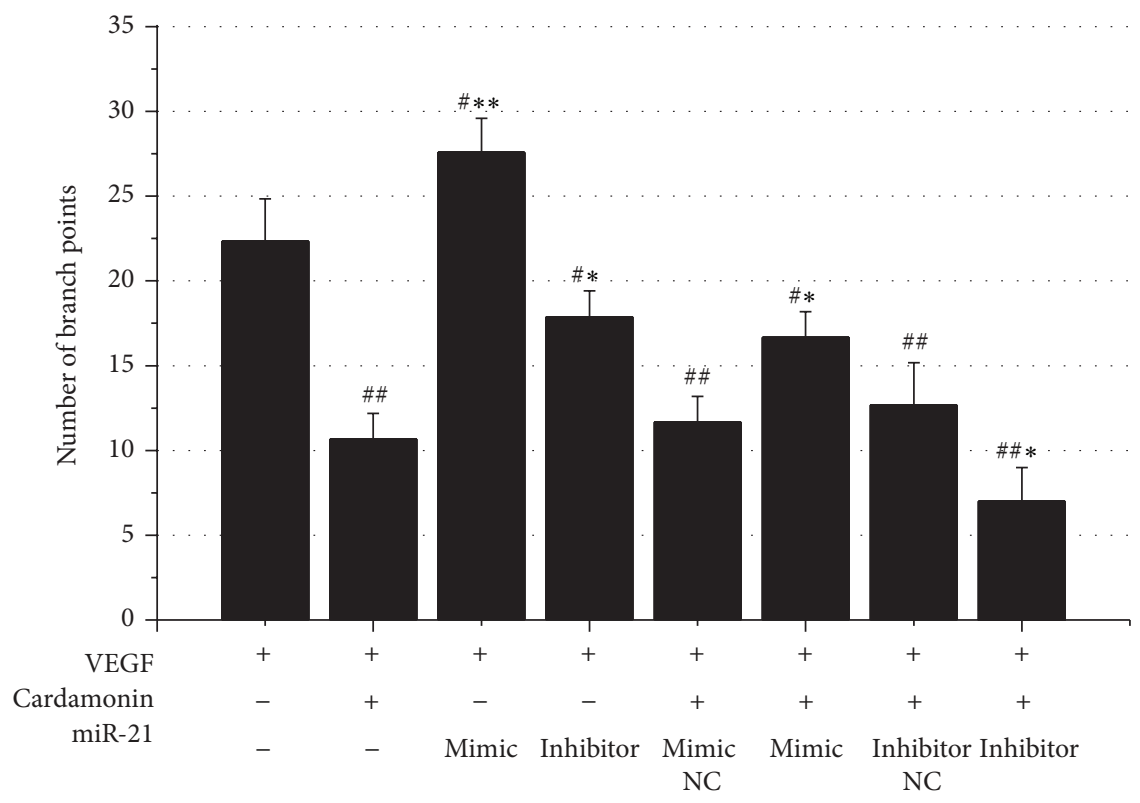

(b)

FIGURE 4: Cardamonin-mediated inhibition of HUVECs tube formation. Note: (a) HUVECs in a six-well plate were photographed at the $24 \mathrm{~h}$ time points. (b) The number of branch points after treated with VEGF, VEGF + cardamonin, VEGF + miR-21s, or VEGF + cardamonin + miR-21s. ${ }^{\# \# \# ~} p<0.05$ and 0.01 , respectively, as compared with the VEGF group, ${ }^{*, * *} p<0.05$ and 0.01 , respectively, as compared with the $\mathrm{VEGF}+$ cardamonin group.

migration, and tube-forming capacity of retinal microvascular endothelial cells (RMECs) [30]. Zhao et al. evidenced that miR-21 can mediate arsenite induced HUVEC upregulation VEGF and promote angiogenesis [31]. In our present study, we found VEGF could upregulate miR-21 expression, and overexpression miR-21 could significantly enhance VEGFinduced HUVEC migration and tube formation, which confirmed the importance of miR-21 on angiogenesis.

Concerning the mechanism of cardamonin on antiangiogenesis, miR-21 mimics and inhibitors were transfected on HUVEC. Results indicated that treatment with miR21 mimics abrogated the cardamonin-mediated inhibition of HUVEC proliferation, migration, and angiogenesis, while treatment with miR-21 inhibitors aggravated the cardamonin-mediated inhibition of HUVEC proliferation, migration, and angiogenesis, indicating that miR-21 might play a vital role in cardamonin-induced inhibition of angiogenesis triggered by VEGF.

It is noted that other miRNAs, for example, miR-132 and miR-16, were also obviously downregulated after treatment 
with $50 \mu \mathrm{M}$ cardamonin. It is reported that miR-132 plays an important role in angiogenesis in infectious ocular disease [32] and miR-16 affects the angiogenesis by targeting VEGF $[33,34]$. Therefore, their roles in regulation of cardamonininduced antiangiogenesis should be addressed in future studies.

Our previous study showed that cardamonin decreased the phosphorylation of ERK and AKT induced by VEGF with dose-dependent manner in HUVECs [14]. It is suggested that miR-21 induces angiogenesis and promotes carcinoma progression through AKT and ERK pathways [35, 36]. Thus, the interactions between miR-21 and AKT and ERK pathways require further study.

\section{Conflict of Interests}

The authors declare that there is no conflict of interests regarding the publication of this paper.

\section{Acknowledgments}

This work was supported by the National Natural Science Foundation of China (81303258), Foundation of Medical Scientific Research Foundation of Zhejiang Province, China (2012KYB136), China Postdoctoral Science Foundation (2014M550335, 2014M561794), and Postdoctoral Science Foundation of Zhejiang Province (BSH1402069). The authors extremely thank Dr. Hua Zhang from University of North Carolina at Chapel Hill for his advises.

\section{References}

[1] X. X. Zhu, W. P. Li, Y. Yu, F. S. Jiang, and Z. S. Ding, “Total flavonoids preparation of the Carya cathayensis Sarg leaves," Chinese Archives of Traditional Chinese Medicine, vol. 31, no. 1, pp. 147-149, 2013.

[2] S. X. Xu, "Study on the extraction technology of flavonoids from G. biloba leaves," Journal of Anhui Agriculture Science, vol. 40, no. 2, pp. 706-707, 2012.

[3] Z. H. Pan, Z. Z. Ye, and S. M. Cui, "Study on extraction process of total flavonoids from Gynostemma pentaphyllum," Journal of Guangdong Pharmaceutical University, vol. 28, no. 1, pp. 44-46, 2012.

[4] N. K. Sahu, S. S. Balbhadra, J. Choudhary, and D. V. Kohli, "Exploring pharmacological significance of chalcone scaffold: a review," Current Medicinal Chemistry, vol. 19, no. 2, pp. 209-225, 2012.

[5] L. M. Ni, C. Q. Meng, and J. A. Sikorski, "Recent advances in therapeutic chalcones," Expert Opinion on Therapeutic Patents, vol. 14, no. 12, pp. 1669-1691, 2004.

[6] M. Y. Lee, C. S. Seo, J. A. Lee et al., "Alpinia katsumadai HAYATA seed extract inhibit LPS-induced inflammation by induction of heme oxygenase-1 in RAW264.7 cells," Inflammation, vol. 35, no. 2, pp. 746-757, 2012.

[7] S. Hatziieremia, A. I. Gray, V. A. Ferro, A. Paul, and R. Plevin, "The effects of cardamonin on lipopolysaccharide-induced inflammatory protein production and MAP kinase and NF $\kappa \mathrm{B}$ signalling pathways in monocytes/macrophages," British Journal of Pharmacology, vol. 149, no. 2, pp. 188-198, 2006.

[8] E. Lin, W.-H. Lin, S.-Y. Wang et al., "Flavokawain B inhibits growth of human squamous carcinoma cells: involvement of apoptosis and cell cycle dysregulation in vitro and in vivo," The Journal of Nutritional Biochemistry, vol. 23, no. 4, pp. 368-378, 2012.

[9] S. P. Bajgai, V. Prachyawarakorn, C. Mahidol, S. Ruchirawat, and P. Kittakoop, "Hybrid flavan-chalcones, aromatase and lipoxygenase inhibitors, from Desmos cochinchinensis," Phytochemistry, vol. 72, no. 16, pp. 2062-2067, 2011.

[10] S. Tewtrakul, S. Subhadhirasakul, J. Puripattanavong, and T. Panphadung, "HIV-1 protease inhibitory substances from the rhizomes of Boesenbergia pandurata Holtt," Songklanakarin Journal of Science and Technology, vol. 25, no. 6, pp. 504-508, 2003.

[11] Q. Liao, D.-H. Shi, W. Zheng, X.-J. Xu, and Y.-H. Yu, “Antiproliferation of cardamonin is involved in mTOR on aortic smooth muscle cells in high fructose-induced insulin resistance rats," European Journal of Pharmacology, vol. 641, no. 2-3, pp. 179-186, 2010.

[12] Y.-J. Shen, X.-X. Zhu, X. Yang et al., "Cardamonin inhibits angiotensin II-induced vascular smooth muscle cell proliferation and migration by downregulating p38 MAPK, Akt, and ERK phosphorylation," Journal of Natural Medicines, vol. 68, no. 3, pp. 623-629, 2014.

[13] Z. Wei, J. Yang, Y.-F. Xia, W.-Z. Huang, Z.-T. Wang, and Y. Dai, "Cardamonin protects septic mice from acute lung injury by preventing endothelial barrier dysfunction," Journal of Biochemical and Molecular Toxicology, vol. 26, no. 7, pp. 282-290, 2012.

[14] S.-S. Tian, F.-S. Jiang, K. Zhang et al., "Flavonoids from the leaves of Carya cathayensis Sarg. inhibit vascular endothelial growth factor-induced angiogenesis," Fitoterapia, vol. 92, no. 1, pp. 34-40, 2013.

[15] L. Varinska, P. Gal, G. Mojzisova, L. Mirossay, and J. Mojzis, "Soy and breast cancer: focus on angiogenesis," International Journal of Molecular Sciences, vol. 16, no. 5, pp. 11728-11749, 2015.

[16] G. Azizi, R. Boghozian, and A. Mirshafiey, "The potential role of angiogenic factors in rheumatoid arthritis," International Journal of Rheumatic Diseases, vol. 17, no. 4, pp. 369-383, 2014.

[17] P. M. Winter, A. M. Neubauer, S. D. Caruthers et al., "Endothelial alpha(v)beta3 integrin-targeted fumagillin nanoparticles inhibit angiogenesis in atherosclerosis," Arteriosclerosis, Thrombosis, and Vascular Biology, vol. 26, no. 9, pp. 2103-2109, 2006.

[18] G. Sotiropoulou, G. Pampalakis, E. Lianidou, and Z. Mourelatos, "Emerging roles of microRNAs as molecular switches in the integrated circuit of the cancer cell," $R N A$, vol. 15, no. 8, pp. 1443-1461, 2009.

[19] Y. Zhao and D. Srivastava, "A developmental view of microRNA function," Trends in Biochemical Sciences, vol. 32, no. 4, pp. 189197, 2007.

[20] A. Eulalio, E. Huntzinger, and E. Izaurralde, "Getting to the root of miRNA-mediated gene silencing," Cell, vol. 132, no. 1, pp. 9$14,2008$.

[21] S. Wang and E. N. Olson, "AngiomiRs-key regulators of angiogenesis," Current Opinion in Genetics \& Development, vol. 19, no. 3, pp. 205-211, 2009.

[22] C. Urbich, A. Kuehbacher, and S. Dimmeler, "Role of microRNAs in vascular diseases, inflammation, and angiogenesis," Cardiovascular Research, vol. 79, no. 4, pp. 581-588, 2008.

[23] L.-J. Chen, S. H. Lim, Y.-T. Yeh, S.-C. Lien, and J.-J. Chiu, "Roles of microRNAs in atherosclerosis and restenosis," Journal of Biomedical Science, vol. 19, no. 1, article 79, 2012.

[24] S. Patan, "Vasculogenesis and angiogenesis," Cancer Treatment and Research, vol. 117, no. 2, pp. 3-32, 2004. 
[25] J.-L. Gao, X. Ji, T.-C. He et al., "Tetrandrine suppresses cancer angiogenesis and metastasis in 4T1 tumor bearing mice," Evidence-Based Complementary and Alternative Medicine, vol. 2013, Article ID 265061, 12 pages, 2013.

[26] P. Nyberg, L. Xie, and R. Kalluri, "Endogenous inhibitors of angiogenesis," Cancer Research, vol. 65, no. 10, pp. 3967-3979, 2005.

[27] H. Y. Hu, Y. M. Li, J. Y. Gu et al., "Antisense oligonucleotide against miR-21 inhibits migration and induces apoptosis in leukemic K562 cells," Leukemia \& Lymphoma, vol. 51, no. 4, pp. 694-701, 2010.

[28] T. Moriyama, K. Ohuchida, K. Mizumoto et al., "MicroRNA21 modulates biological functions of pancreatic cancer cells including their proliferation, invasion, and chemoresistance," Molecular Cancer Therapeutics, vol. 8, no. 5, pp. 1067-1074, 2009.

[29] S. M. Zhu, H. L. Wu, F. T. Wu, D. Nie, S. Sheng, and Y.-Y. Mo, "MicroRNA-21 targets tumor suppressor genes in invasion and metastasis," Cell Research, vol. 18, no. 3, pp. 350-359, 2008.

[30] J. Guduric-Fuchs, A. O'Connor, A. Cullen et al., "Deep sequencing reveals predominant expression of miR-21 amongst the small non-coding RNAs in retinal microvascular endothelial cells," Journal of Cellular Biochemistry, vol. 113, no. 6, pp. 20982111, 2012.

[31] Y. Zhao, Y. Xu, F. Luo et al., "Angiogenesis, mediated by miR-21, is involved arsenite-induced carcinogenesis," Toxicology Letters, vol. 223, no. 1, pp. 35-41, 2013.

[32] S. Mulik, J. Xu, P. B. J. Reddy et al., "Role of miR-132 in angiogenesis after ocular infection with herpes simplex virus," American Journal of Pathology, vol. 181, no. 2, pp. 525-534, 2012.

[33] J.-K. Lee, S.-R. Park, B.-K. Jung et al., "Exosomes derived from mesenchymal stem cells suppress angiogenesis by downregulating VEGF expression in breast cancer cells," PLoS ONE, vol. 8, no. 12, Article ID e84256, 2013.

[34] C.-Y. Sun, X.-M. She, Y. Qin et al., "miR-15a and miR-16 affect the angiogenesis of multiple myeloma by targeting VEGF," Carcinogenesis, vol. 34, no. 2, pp. 426-435, 2013.

[35] L.-Z. Liu, C.-Y. Li, Q. Chen et al., "Mir-21 induced angiogenesis through AKT and ERK activation and HIF-1 $\alpha$ expression," PLoS ONE, vol. 6, no. 4, Article ID e19139, 2011.

[36] L. L. Bao, Y. Yan, C. Xu et al., "MicroRNA-21 suppresses PTEN and hSulf-1 expression and promotes hepatocellular carcinoma progression through AKT/ERK pathways,' Cancer Letters, vol. 337, no. 2, pp. 226-236, 2013. 

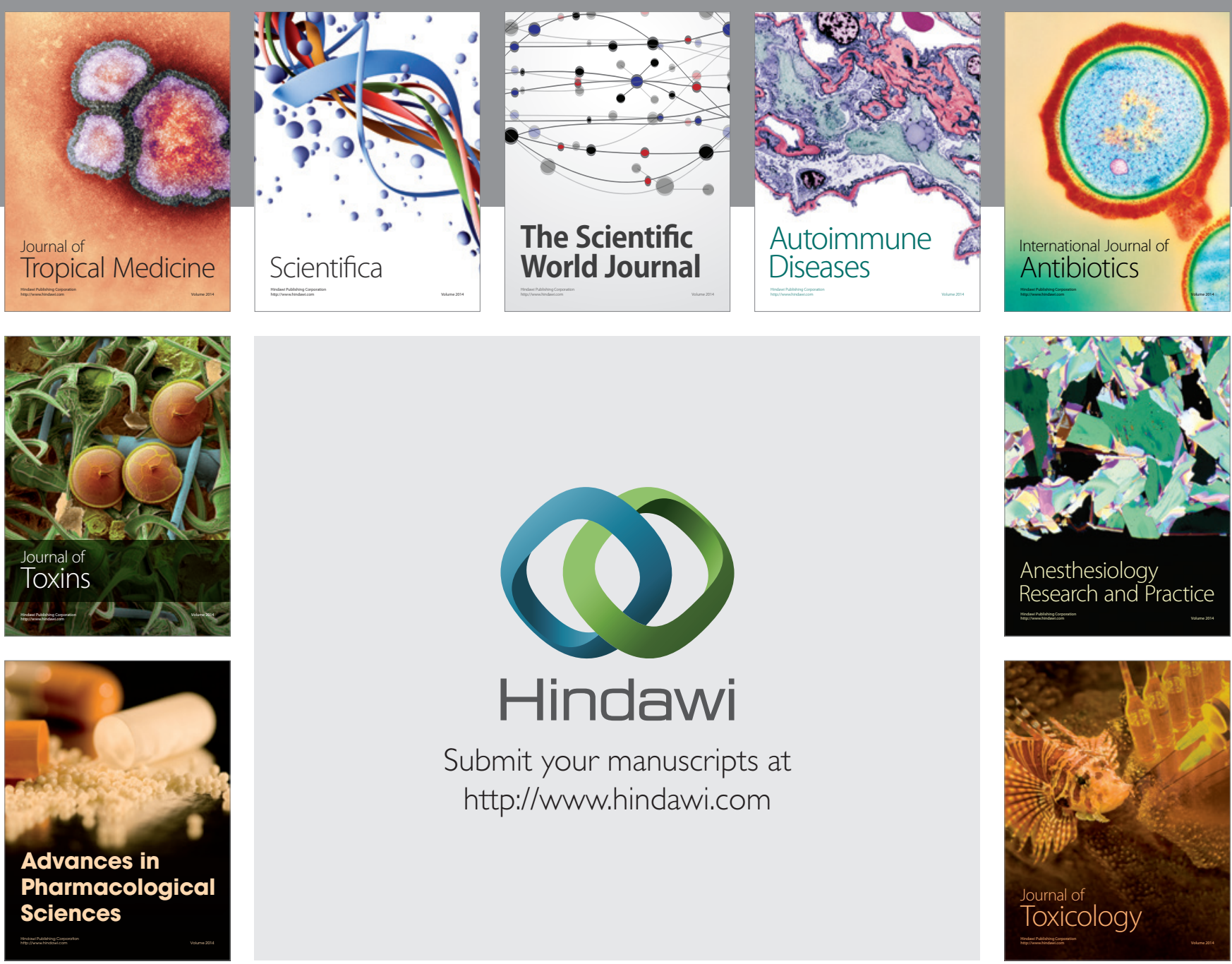

\section{Hindawi}

Submit your manuscripts at

http://www.hindawi.com
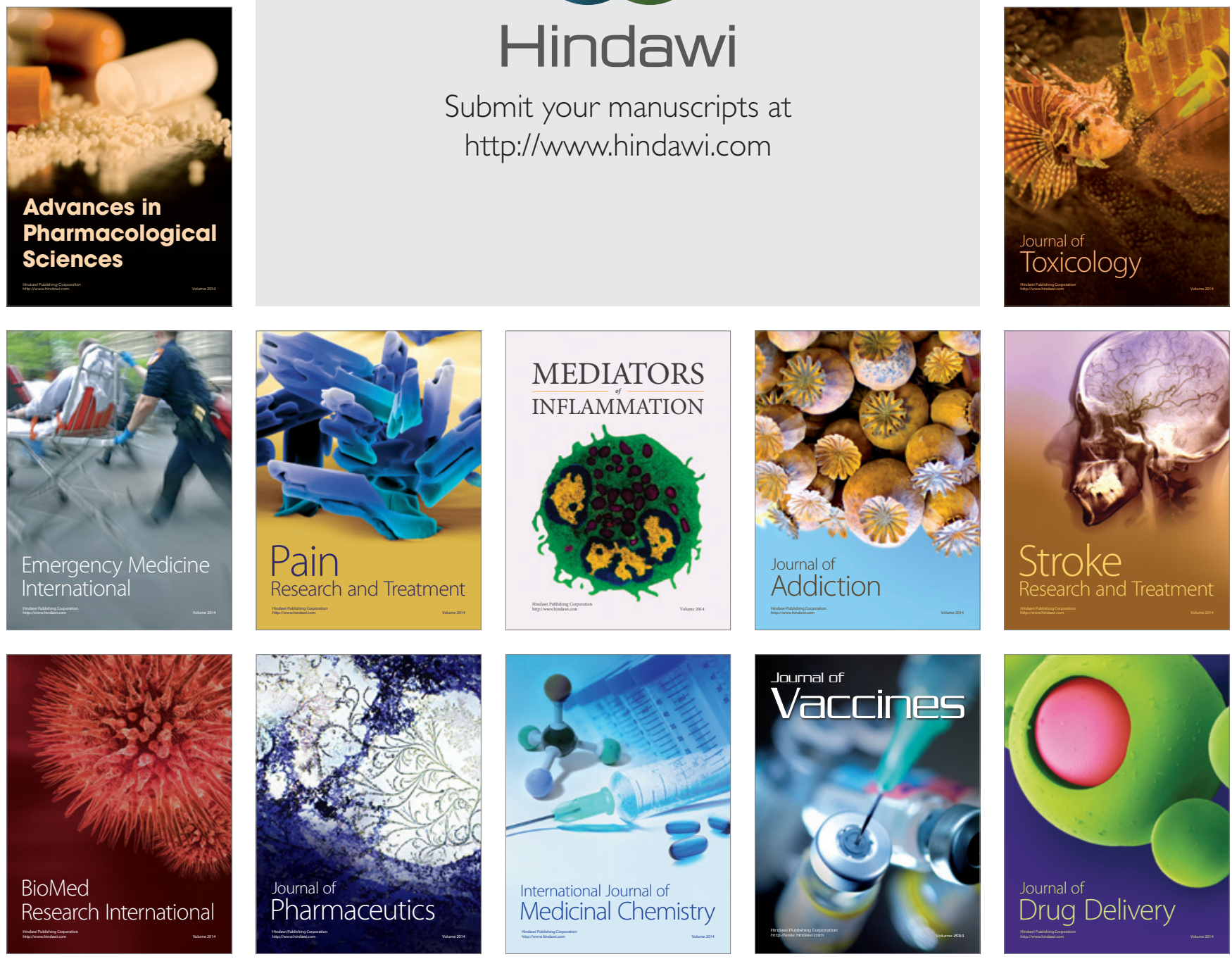